\title{
The Role of Medical Image Computing and Machine Learning in Healthcare
}

\author{
Frederik Maes, David Robben, Dirk Vandermeulen, and Paul Suetens
}

\begin{abstract}
Medical image computing aims at developing computational strategies for robust, automated, quantitative analysis of relevant information from medical imaging data in order to support diagnosis, therapy planning and follow-up, and biomedical research. Medical image analysis is complicated by the complexity of the data itself - involving 3D tomographic images acquired with different modalities that are based on different physical principles, each with their own intrinsic characteristics and limitations, and by the complexity of the scene - involving normal and pathological anatomy and function, with complex 3D shapes and significant inter-subject variability. Hence, model-based approaches are needed that take prior knowledge about the image appearance of the relevant objects in the scene into account. These models are parameterized to deal with variability in object appearance, such that the image analysis problem can be formulated as an optimization problem of finding the model parameters that best explain the image data. Depending on the representation chosen for the model, different approaches can be discriminated. Machine learning offers the possibility to learn suitable models from previously analyzed data itself.
\end{abstract}

Key words: medical imaging, registration, segmentation, visualization, modeling, validation

\section{Introduction}

Due to continuing technological advances in medical image acquisition, novel imaging modalities are being introduced in medical practice, such as multi-slice (volumetric) and multi-energy CT, multi-parametric and multi-frame (dynamic) MRI,

Frederik Maes · David Robben · Dirk Vandermeulen · Paul Suetens

KU Leuven, Dept. ESAT/PSI, Kasteelpark Arenberg 10 bus 2441, 3000 Leuven, Belgium e-mail:

frederik.maes@kuleuven.be 
multi-dimensional (3D+time) US, multi-planar interventional imaging, or multimodal (hybrid) PET/CT and PET/MRI imaging technologies [1]. The analysis of the large amounts of imaging data created by these modalities has become a tremendous challenge and a real bottleneck for diagnosis, therapy planning and follow-up, and biomedical research. At the same time, the general adoption of digital picture archiving and communication systems (PACS) in radiology, and their integration within the overall hospital information system, makes that large databases of medical images and associated relevant medical information of patients (including for instance demographics, clinical findings, blood tests, pathology, genomics, proteomics) are being built up. It is to be expected that such databases will become more and more accessible for research purposes, provided that technical challenges and privacy issues can be properly dealt with. The availability of well-documented medical imaging "big data" offers new opportunities for groupwise analyses within specific subject groups, such as characterization of normal and abnormal variation between subjects and detection of individual patient anomalies (computer-aided diagnosis), discovery of early markers of disease onset and progression (imaging biomarkers), optimal therapy selection and prediction of therapy outcome (radiomics in radiotherapy), and correlation of genotype and phenotype related findings (imaging genetics). In order to optimally exploit all available imaging data and to support the effective use of "big data" involving medical images in the context of personalized medicine, reliable computer-aided image analysis becomes indispensable to extract and quantify the relevant information from the imaging data, to fuse complementary information and to support the interpretation thereof.

\section{Medical image analysis}

Medical image analysis involves measurements in medical images, i.e. the extraction of relevant quantitative information from the images. Manual measurements by human experts in large 3D medical imaging datasets (in particular by radiologists in clinical practice) are not only tedious and time-consuming and thus impractical in clinical routine, but also subject to significant intra- and inter-observer variability, which undermines the significance of the clinical findings derived from them. There is, therefore, great need for more efficient, reliable and well-validated automated or semi-automated methods for medical image analysis to enable computer-aided image interpretation in routine clinical practice in a large number of applications. Which information needs to be quantified from the images, is of-course highly application specific. While many applications in computer vision involve the detection or recognition of an object in an image, whereby the precise geometry of the objects is often not relevant (e.g. image classification, object recognition) or may be known a priori (e.g. machine vision), medical image analysis often concerns the quantification of specific geometric features of the objects of interest (e.g. their position, extent, size, volume, shape, symmetry...), the assessment of anatomical changes over time (e.g. organ motion, tissue deformation, growth, lesion evolution, 
atrophy, ageing...) or the detection and characterization of morphological variation between subjects (e.g. normal versus abnormal development, genotype related variability, pathology...). The analysis of 3D shape and shape variability of anatomical objects in images is thus a fundamental problem in medical image analysis. Apart from morphometry, quantification of local or regional contrast or contrast differences is of interest in many applications, in particular in functional imaging, such as fMRI, PET or MR diffusion and perfusion imaging.

Within the wide variety of medical imaging applications, most image analysis problems involve a combination of the following basic tasks [2].

\subsection{Image segmentation}

Image segmentation involves the detection of the objects of interest in the image and defining their boundaries, i.e. discriminating between the image voxels that belong to a particular object and those that do not belong to the object. Image segmentation is a prerequisite for quantification of the geometric properties of the object, in particular its volume or shape. Image segmentation can be performed in different ways: boundary-wise by delineating the contour or surface of the object in one (2D) or multiple (3D) image slices; region-wise by grouping voxels that are likely to belong to the same object into one or multiple regions; or voxel-wise by assigning each voxel in the image as belonging to a particular object, tissue class or background. Class labels assigned to a voxel can be probabilistic, resulting in a soft or fuzzy segmentation of the image. Accurate 3D segmentation of complex shaped objects in medical images is usually complicated by the limited resolution of the images (leading to loss of detail and contrast due to partial volume artefacts) and by the fact that the resolution if often not isotropic (mostly multi-slice 2D instead of truly 3D acquisitions). Hence, interpolation is usually needed to fill in the missing information in the data. In clinical practice, precise 3D measurements (e.g. volumetry) may be too tedious and time consuming, such that often a simplified, approximate $2 \mathrm{D}$ or $1 \mathrm{D}$ analysis is used instead (e.g. for estimation of lesion size).

\subsection{Image registration}

Image registration involves determining the spatial relationship between different images, i.e. establishing spatial correspondences between images or image matching, in particular based on the image content itself [3]. Different images acquired at different time points (e.g. before and after treatment), or with different modalities (e.g. CT, MRI, PET brain images), or even from different subjects (e.g. diseased versus healthy) often contain complementary information that has to be fused and 
analysed jointly, preferably at the voxel level to make use of the full resolution of the images. Image registration is needed to compensate for a priori unknown differences in patient positioning in the scanner, for organ or tissue deformations between different time points, or for anatomical variation between subjects. After proper registration, the images can be resampled onto a common geometric space and fused, i.e. spatially corresponding voxels can be precisely overlaid, which drastically facilitates the joint analysis of the images. In some cases, when deformations are ignorable, the registration solution can be represented as an affine transformation matrix with a small number of parameters, but in general a more complex transformation in the form of a locally flexible deformation field is needed to accommodate for non-rigid distortions between the images.

\subsection{Image visualization}

The information that is extracted from the images ideally needs to be presented in the most optimal way to support diagnosis and therapy planning, i.e. such that the correct interpretation by the user of all relevant image data is maximally facilitated for a specific application. For 3D medical images, 2D multi-planar visualization is not well suited to assess structural relationships within and between objects in $3 \mathrm{D}$, for which true 3D visualization approaches are to be preferred. To this end, either surface rendering or volume rendering can be applied. Surface rendering assumes that a 3D segmentation of the objects of interest is available and renders these within a 3D scene under specified lighting conditions (e.g. ambient light, point light sources) by assigning material properties to each surface or surface element, that specify its specular and diffuse light reflection, transmission, scattering... Volume rendering instead renders the image voxels directly by specifying suitable transfer functions that assign each voxel a color and opacity depending on their intensity. While in principle volume rendering does not require a prior segmentation of the objects of interest, in practice a prior segmentation of the image is often applied such that the transfer functions can be made spatially dependent and object specific, which allows to discriminate between voxels with similar intensity belonging to different objects. In clinical applications such as image-based surgery planning or image-guided intra-operative navigation, additional tools need to be provided to manipulate the objects in the 3D scene (e.g. cutting), to add virtual objects to the scene (e.g. implants), or to fuse the virtual reality scene with real-world images (e.g. intra-operative images). While such augmented reality techniques can improve the integrated presentation of all available information during an intervention (e.g. using a head-mounted display), their introduction in clinical practice is far from trivial.

Image segmentation, registration and visualization should not be seen as separate subproblems in medical image analysis that can be addressed independently, each using a specific set of strategies. On the contrary, they are usually intertwined and 
an optimal solution for a particular image analysis problem can only be achieved by considering segmentation, registration and visualization jointly. For instance, image registration can be used as a computational strategy for image segmentation by matching the image to be segmented to a similar image (e.g. from a different patient, or an atlas template) that has been previously segmented (i.e. atlas-based segmentation). Vice versa, image registration can benefit from the fact that a prior segmentation of similar structures in each image is already available, as these provides strong clues to guide the registration process. Joint visualization of multiple different images, acquired for instance pre-operatively and intra-operatively, requires that registration issues between all images residing in different coordinate systems have been resolved. This in turn is facilitated when suitable visualization and manipulation tools are available to verify and adjust the registration interactively by visual feedback (i.e. visual matching). Moreover, in applications involving image-guided treatment, the pre-operative treatment plan needs to be transferred onto the patient during the intervention and the intra-operative position of the instruments needs to be tracked in the images. This registration problem typically requires additional hardware to be installed in the treatment room (e.g. an optical tracking system).

\section{Challenges}

Medical image analysis is complicated by different factors, in particular the complexity of the data, the complexity of the objects of interest, and the complex validation.

\subsection{Complexity of the data}

Medical images are typically 3D tomographic images. The 3D nature of the images provides additional information, but also an additional dimension of complexity. Instead of processing the data in $2 \mathrm{D}$ slice by slice, $3 \mathrm{D}$ processing is usually more effective as it allows to take spatial relationships in all three dimensions into account, provided that the resolution of the data in-plane and out-plane is comparable. Medical images are based on different physical principles and the quantification of the images is complicated by the ambiguity that is induced by the intrinsic limitations of the image acquisition process, in particular limited resolution, lack of contrast, noise, and the presence of artifacts. Moreover, many applications involve the analysis of complementary information provided by multiple images, for instance to correlate anatomical and functional information, to assess changes over time or differences between subjects. It is clear that the variable, multi- $X$ nature of the images to be analyzed (multi-dimensional, multi-modal, multi-temporal, multi-parametric, multi-subject, multi-center) poses specific challenges. 


\subsection{Complexity of the objects of interest}

The objects of interest in medical images are typically anatomical structures (sometimes also other structures, e.g. implants), either normal or pathological (e.g. lesions), that can be rigid (e.g. bony structures) or flexible to some extent (e.g. soft tissue organs). Anatomical structures may exhibit complex shape, such as the cortical surface of the brain, the cerebral and coronary vessels, or the bronchial tree in the lung. Such complex shapes can not easily be described by a mathematical model. Moreover, anatomical structures can show large intra-subject shape variability, due to internal soft tissue deformations (e.g. breathing-related motion, bowel activity), as well as inter-subject variability, due to normal biological variation and pathological changes. In general, the appearance of similar structures in different images (of the same subject at different time points or from different subjects) can show significant variability, both in shape as in intensity. Computational strategies for medical image analysis need to take this variability into account and be sufficiently robust to perform well under a variety of conditions.

\subsection{Complexity of the validation}

Medical image analysis involves the quantification of internal structures of interest in real-world clinical images that are not readily accessible from the outside. Hence, assessment of absolute accuracy is often impossible in most applications, due to lack of ground truth. As an alternative, a known hardware phantom that mimics the relevant objects of interest could be imaged, but the realism of such a phantom compared to the actual in-vivo situation is often questionable. Moreover, a hardware phantom usually constitutes a fairly rigid design that is not well apt to be adapted to different variable anatomical instances. Instead, the use of a software phantom in combination with a computational tool that generates simulated images based on a model of the imaging process provides more flexibility, both with respect to the imaged scene as the image acquisition setup itself. But again, such simulated images often fail to capture the full complexity of real data. In clinical practice, ground truth is typically provided by manual analysis by a clinical expert, for instance manual delineation in case of image segmentation or manual annotation of corresponding anatomical landmarks in case of image registration. As already mentioned, such manual analysis is subject to intra- and inter-observer variability, which should be taken into account when validating (semi-)automated methods. Apart from accuracy, precision, consistency and robustness of the method are to be considered as well when evaluating its clinical usability. 


\section{Medical image computing}

Medical image computing, which is a branch of scientific computing at the intersection of medical imaging, computer vision and machine learning, aims at developing computational strategies for medical image analysis that can cope with the complexity of medical imaging data to enable (semi-)automated analysis with sufficient accuracy and robustness. Such strategies rely on mathematical models that incorporate prior knowledge about the typical appearance of the objects of interest in the images, including photometric properties (e.g. intensity, contrast, texture), geometric properties (e.g. position, shape, motion) and context (e.g. relations to other objects) [4]. Model-based image analysis involves the construction of an appropriate parameterized representation for the model, the derivation of an objective function for assessing the goodness of fit of the model to the data, and the selection of a suitable optimization strategy for finding the optimal parameters of the model instance that best fits the image data. The models need to be sufficiently flexible to account for image appearance variations, due to e.g. variability in the image acquisition itself, normal biological shape variability, motion and deformation, and pathology. The flexibility of the model is determined by the specific representation that is chosen for the model, its parameterization and number of degrees of freedom, and by the constraints imposed on its parameters.

Simplistic models based on generic, heuristic assumptions about the appearance of the objects in the images, for instance about their intensity homogeneity and (dis)similarity or their boundary continuity and smoothness, are in general (not suited for medical image analysis applications, as they are not powerful enough to capture the full complexity of the problem apart from few exceptions, such as segmentation of bony structures in CT). Instead, more sophisticated approaches are needed that incorporate application-specific information about the images to be analysed. A natural and powerful strategy is to construct suitable models from the data itself by analysis of a representative set of previously analyzed images. Such statistical models could in principle ensure that the degrees of freedom of the model are optimally tuned to the relevant variation within the data of each specific application, provided that the training set of previously analyzed images from which the model is constructed is large enough and representative for the population of subjects being considered. Instead of making use of a generic parameterization of the model that is applicable to many different applications, the construction of application-specific statistical models allows to decrease the number of relevant parameters of the model by exploiting correlations in the data, for instance by adopting a multi-variate Gaussian model for the underlying distribution of the data or by using dimensionality reduction techniques such as principal component analysis. Instead of postulating a specific analytical form for the statistical model, more general supervised data-driven approaches can also be used to infer the relationship between a vector of specific features extracted from the data and the desired quantification outcome. To this end, various machine learning strategies for feature-based classification, such as support vector machines or random decision forests, can be used [5]. 
Recent advances in supervised learning of models from training data, especially deep learning based on convolutional neural networks, have shown great promise for many problems in computer vision, including image classification, object recognition and segmentation. Deep learning also shows great promise for medical imaging applications [6]. The analysis problem is formulated as a classification task based on a large set of non-specified local features that are assumed to be homogeneous within and between all images separately and that are to be learned by the neural network itself. Neural networks define highly complex function classes and large amounts of data are typically necessary for them to converge to a stable solution with good generalization ability. This requirement is usually not met in medical image analysis, where the availability of training data is limited, which poses additional challenges that are not addressed in the majority of the machine learning literature. Moreover, the analysis of shape and shape variability, which is a fundamental problem in medical image analysis, typically includes dispersed non-local patterns derived from dense spatial correspondences between heterogeneous images analyzed jointly, for which it is not evident how this problem could be formulated as a classification problem using current neural network architectures. In addition, model-based analysis aims at avoiding heuristics and implicit assumptions about the data as much as possible by making such assumptions explicit using a parametric model. While deep learning seems to comply with this paradigm by avoiding heuristic feature construction and selection, plenty of heuristics are embedded in the actual implementation of the chosen neural network architecture, in the optimization strategy and in the sampling of the training data presented to the network, which complicates the interpretation of the model and the optimization of its performance.

\section{Model-based image analysis}

Model-based image analysis makes use of a model of the image appearance of the objects of interest in the images. The model represents prior knowledge about the geometric, photometric and contextual properties of the objects of interest in the images. The model should be sufficiently specific to deal with ambiguity in the imaging data and at the same time sufficiently flexible to accommodate for normal biological shape variability, pathological changes or non-rigid deformations of the objects of interest. The model is fitted to the image data using a suitable measure for the goodness of fit. This can be generally formulated as follows. Let $I$ be the image data and $M(\theta)$ the representation of the model with parameters $\theta$. Fitting the model $M(\theta)$ to the image data $I$ involves finding the model instance $M\left(\theta^{*}\right)$ with parameters $\theta^{*}$ for which the a posteriori probability $\operatorname{Prob}(M(\theta) \mid I)$ is maximized:

$$
\theta^{*}=\arg \max _{\theta} \operatorname{Prob}(M(\theta) \mid I)
$$

Using Bayes' rule, the a posteriori probability can be written as 


$$
\operatorname{Prob}(M(\theta) \mid I)=\frac{\operatorname{Prob}(I \mid M(\theta)) \cdot \operatorname{Prob}(M(\theta))}{\operatorname{Prob}(I)}
$$

with $\operatorname{Prob}(I \mid M(\theta))$ the likelihood of observing the data $I$ given the model, $\operatorname{Prob}(M(\theta))$ the prior probability of the model instance with parameters $\theta$, and $\operatorname{Prob}(I)$ the probability of observing the data $I$. Because the latter is independent of $M(\theta)$, it follows that the optimal model parameters should satisfy

$$
\theta^{*}=\arg \max _{\theta}(\operatorname{Prob}(I \mid M(\theta)) \cdot \operatorname{Prob}(M(\theta)))
$$

\subsection{Energy minimization}

Instead of estimating and maximizing $\operatorname{Prob}(M(\theta) \mid I)$ directly, the model fitting can as well be performed by estimating the prior probability $\operatorname{Prob}(M(\theta))$ and the data likelihood $\operatorname{Prob}(I \mid M(\theta))$ and maximizing their product. The maximum is preserved by taking the logarithm of the right-hand side (as the logarithm is monotonically increasing):

$$
\begin{aligned}
\theta^{*} & =\arg \max _{\theta} \log (\operatorname{Prob}(I \mid M(\theta)) \cdot \operatorname{Prob}(M(\theta))) \\
& =\arg \max _{\theta} \log (\operatorname{Prob}(I \mid M(\theta)))+\log (\operatorname{Prob}(M(\theta)))
\end{aligned}
$$

By adopting a Gibbs distribution for both the prior and the data likelihood, this optimization problem can be formulated as energy minimization:

$$
\begin{gathered}
\operatorname{Prob}(M(\theta))=\frac{\exp \left(-E_{\text {int }}(\theta)\right)}{Z_{\text {int }}} \\
\operatorname{Prob}(I \mid M(\theta))=\frac{\exp \left(-E_{\text {ext }}(\theta \mid I)\right)}{Z_{\text {ext }}(I)}
\end{gathered}
$$

with $Z_{\text {int }}$ and $Z_{\text {ext }}(I)$ normalization constants (independent of $\theta$ ) and $E_{\text {int }}$ and $E_{\text {ext }}$ the internal and external energy respectively, such that

$$
\begin{aligned}
\theta^{*} & =\arg \max _{\theta} \log \left(\frac{\exp \left(-E_{\text {int }}(\theta)\right)}{Z_{\text {int }}}\right)+\log \left(\frac{\exp \left(-E_{\text {ext }}(\theta \mid I)\right)}{Z_{\text {ext }}}\right) \\
& =\arg \min _{\theta}\left(E_{\text {int }}(\theta)+E_{\text {ext }}(\theta \mid I)\right)
\end{aligned}
$$

The internal energy $E_{\text {int }}$ of the model instance $\theta$ is a measure for its a priori likelihood, while the external energy $E_{\text {ext }}$ represents the goodness of fit of the model instance $\theta$ to the data. Note that $E_{\text {ext }}$ depends on the image data $I$, while $E_{\text {int }}$ is independent of $I$. The actual formulation of the energy terms in the objective function $E$ depends on the choice of the representation for the model $M(\theta)$ and its param- 
eterization and on the specific prior knowledge about the appearance of the object of interest in the images that is to be captured by the model. In practice, the energy minimization formalism is very versatile and makes it easy to implement various different deterministic or statistical, purely mathematical or biomechanically inspired, heuristic or learned constraints (or penalties) on the model [7].

In practice, both energy terms are not absolute but relative and need to be weighted with respect to each other, which can be made explicit by introducing a weight $\gamma$ in the energy function:

$$
\begin{aligned}
E(\theta \mid I, \gamma) & =E_{\operatorname{ext}}(\theta \mid I)+\gamma E_{\text {int }}(\theta) \\
\theta^{*} & =\arg \min _{\theta} E(\theta \mid I, \gamma)
\end{aligned}
$$

By tuning the value of $\gamma$, the optimal model instance can be driven towards favoring more data congruency ( $\gamma$ small $)$ or towards more model fidelity ( $\gamma$ large). Additional parameters may be embedded in the definition of $E_{\text {int }}$ and $E_{\text {ext }}$ itself. Tuning of such hyperparameters for optimal performance for the given application is an intrinsic difficulty of any model-based image analysis approach.

The solution of this optimization problem requires a suitable optimization strategy. Typically, this can be done using variational calculus and iterative gradient descent on $E$ w.r.t. the model parameters $\theta$, starting from a suitable initialization $\theta_{0}$ that is sufficiently close to the actual optimal value $\theta^{*}$. In practice, such local search procedure yields a local optimum $\theta^{+}$that is not guaranteed to be globally optimal and that depends on the initialization and on parameters of the optimization procedure itself (e.g. step size, stop criterion). Alternatively, by discretizing the solution space $\theta$, the problem can be formulated as a discrete optimization problem on a graph, for which efficient global optimization strategies are available, such as dynamic programming or min cut/max flow algorithms.

\subsection{Classification / regression}

For the energy function $E(\theta \mid I, \gamma)$ to be relevant and sufficiently informative for a specific image analysis problem, realistic and sufficiently sophisticated formulations of the data likelihood $\operatorname{Prob}(I \mid M(\theta))$ and the prior $\operatorname{Prob}(M(\theta))$ are needed. These can in principle be derived by statistical analysis of a set of similar, previously analyzed images $\mathscr{T}=\left\{\left(I_{i}, \theta_{i}\right), i=1 \ldots n\right\}$. In that case, a direct estimate $\mathscr{P}(\theta \mid I, \mathscr{T})$ of the posterior probability $\operatorname{Prob}(M(\theta) \mid I)$ may then as well be derived from the training data $\mathscr{T}$, such that the optimal model instance $M\left(\theta^{*}\right)$ may be obtained by maximization of $\mathscr{P}$ :

$$
\theta^{*}=\arg \max _{\theta} \mathscr{P}(\theta \mid I, \mathscr{T})=\Phi(I \mid \mathscr{T})
$$


The function $\Phi$ maps every input $I$ onto the most likely output $\theta^{*}$ based on the given training data $\mathscr{T}$. The output $\theta$ can be defined as a single value for the entire image (e.g. in case of image classification), or voxel-wise (e.g. in case of segmentation). In case $\theta$ takes on discrete values (e.g. object class labels), the function $\Phi$ acts as a classifier, while if $\theta$ takes on continuous values (e.g. coordinates), $\Phi$ acts as a regression function. In practice, the dimensionality of the image $I$ (i.e. the number of voxels) is high, while the number of training samples $n$ is usually much smaller, such that the estimation of $\Phi$ is ill-conditioned. Hence, the problem is often simplified by estimating a function $\Phi_{f}$ based on a limited number of pre-defined features $f=$ $\mathscr{F}(I)$ that are computed from the image $I$ :

$$
\theta_{f}^{*}=\arg \max _{\theta} \mathscr{P}(\theta \mid \mathscr{F}(I), \mathscr{T})=\Phi_{f}(f \mid \mathscr{T})
$$

Different machine learning strategies, such as $k$-nearest neighbours, support vector machines or random forest decision trees, can be applied to construct the mapping $\Phi_{f}$ for a given training set $\mathscr{T}$ and a given set of features $\mathscr{F}$. During training, the parameters $w$ that define the mapping $\Phi_{f}(f \mid w)$, whose representation depends on the chosen learning strategy, are iteratively refined such that estimation performance is maximized on the training set itself, using a measure $L$ (a cost or loss function) that evaluates the difference between the given ground truth $\theta_{i}$ for each image $I_{i}$ in the training set and the estimated $\theta_{i}^{\prime}(w)=\Phi_{f}\left(\mathscr{F}\left(I_{i}\right) \mid w\right)$ :

$$
\begin{aligned}
\mathscr{L}(w \mid \mathscr{T}) & =\sum_{i \in \mathscr{T}} L\left(\theta_{i}, \theta_{i}^{\prime}(w)\right) \\
w^{*} & =\arg \min _{w} \mathscr{L}(w \mid \mathscr{T})
\end{aligned}
$$

By cross-validation against a separate validation set $\mathscr{V}$ of additional instances $\left\{\left(I_{j}, \theta_{j}\right), j=1 \ldots m\right\}$ that are not used for training, the generalization potential of the learned mapping $\Phi_{f}\left(f \mid w^{*}\right)$ to new, previously unseen cases can be assessed. Large differences in performance between training and validation sets $\left(\mathscr{L}\left(w^{*} \mid \mathscr{T}\right)<<\right.$ $\left.\mathscr{L}\left(w^{*} \mid \mathscr{V}\right)\right)$ are an indication that the mapping $\Phi_{f}\left(w^{*}\right)$ is overfitted and that a simpler, less flexible and more regularized mapping would be more appropriate.

Feature vector based classification/regression is a very flexible approach for image analysis, in the sense that multiple, separately computed sets of features $f_{k}$, related to different object properties (e.g. contrast, texture, geometry, context...) or computed from different subparts of the data (e.g. multi-parametric MRI), can be simply combined by concatenating them into an aggregated feature vector $f=$ $\left(f_{1}, f_{2}, \ldots\right)$. Moreover, additional, non-image related features (e.g. clinical parameters, genetical information...) can be easily incorporated in the same way. However, extending the feature vector also increases the dimensionality of the problem and may necessitate proper prior scaling of the different feature ranges, which makes a robust estimation of $\Phi_{f}$ more complex and increases the risk of overfitting. Hence, in practice, a mechanism for optimal feature selection will be typically applied to reduce the dimensionality of the feature space by only maintaining the most relevant features (or combinations thereof) yielding optimal validation performance. 
A drawback of conventional feature vector classification/regression is that the initial set of features $\mathscr{F}(I)$ is pre-defined by the user, which is typically done heuristically and therefore likely suboptimal. Current state-of-the-art machine learning approaches, in particular deep learning using convolutional neural networks with several consecutive hidden layers, alleviate this problem by performing optimal feature computation and selection during training, thus effectively estimating the mapping $\Phi$ directly from the data $I$ itself. Due to the large number of parameters in such networks, different aspects related to network architecture, optimization, regularization, data sampling and augmentation have to be carefully considered.

\section{Computational strategies}

Model-based computational strategies for medical image computing can be broadly classified as either flexible shape fitting or pixel classification.

\subsection{Flexible shape fitting}

Flexible shape fitting makes use of a more or less global parametric model of the image appearance of the object, including photometric, geometric and contextual properties, that is fitted to the actual image data by optimization of an objective function that evaluates the goodness of fit of the model instance. This objective function is often formulated as an energy function and finding the optimal model instance is solved as an energy minimization process. The energy function consists of both external, data-dependent energy terms, which aim at driving the geometry of the model instance to deform towards relevant photometric features of interest in the image, and internal, data-independent energy terms, which serve to constrain the flexibility of the model and account for prior knowledge about the shape of the object. The external energy of the model can be based on local boundary features or more global regional features, and can be defined heuristically, be derived from a statistical modelling of these features, or rely on a specific model of the image acquisition. The internal energy can be based on deterministic penalties imposed on the shape of the model instance (e.g. spatial smoothness), on a (partial or pseudorealistic) biomechanical modelling of the object (e.g. elastic deformation behavior), or on statistical constraints (e.g. the expected shape of the object and its shape variability). Contextual information, for instance the relative position of different objects, can also be accounted for by incorporating cross-terms in the internal energy functions of these objects. Typically, the balance between both energy contributions, i.e. between fidelity to the model and congruency to the data, is controlled by userspecified weights that need to be tuned for each specific application.

The geometry of the model can be represented explicitly, i.e. landmark-based, by representing the model as a set of discrete points defined by their 3D coordinates in 
some suitable space, typically arranged in a graph (a 1D curve, a 2D surface, a 3D mesh, ...), with or without underlying continuous analytical parameterization (e.g. a piece-wice polynomial function). Alternatively, the geometry of the model can be represented implicitly, i.e. image-based, by representing the model as a picture, e.g. a gray value image, label image, probability map, or distance map, whereby the geometry of the model is intrinsically related to the regular spatial grid on which the image is defined. In case of a landmark-based shape model, the shape is altered by modifying the coordinates of the landmarks, either individually or jointly. In case of an image-based shape model, the shape is altered by modifying the intensities of the image, either directly or indirectly, namely by a transformation (affine or non-rigid) of the underlying image grid. Examples of flexible shape fitting strategies using an explicit shape representation include for instance active contours ("snakes") [8], active shape models [10] and active appearance models [11]. Examples of flexible shape fitting strategies using an implicit shape representation include for instance level sets [12], graph cuts [13], eigenfaces [9] and intensity-based non-rigid registration.

While deterministic constraints imposed on the flexibility of the model are necessarily largely heuristic in nature, statistical models aim at avoiding heuristics by learning suitable model constraints from the data itself, based on a representative training set of examples, typically derived from a database of similar images acquired from different subjects (see Figure 1 for an example). A popular strategy for landmark-based statistical shape modeling are point distribution models (PDM) [14]. A PDM is constructed by statistical analysis of the observed variations in the locations of corresponding landmark points defined on all object shapes in a representative training set of shape instances, after appropriate spatial normalization of all shapes to a common coordinate space to eliminate irrelevant, pose-related variability. In practice, the construction of PDMs is complicated by the fact that a sufficiently large training set of previously segmented shape instances needs to be available, which usually involves manual delineation in 3D images, which is tedious and time consuming for a large collection of images, error-prone and subject to intra- and inter-rater variability and uncertainty. Moreover, a sufficiently large set of point correspondences needs to have been established between all shape instances, which by itself is a non-trivial problem for which different strategies have been proposed [15].

In case of image-based models, fitting of the model to new data can be formulated as an image registration problem. Image registration establishes dense spatial correspondences (i.e. a coordinate transformation) between two images based on a suitable local or global similarity measure, such that prior information from one can be propagated onto the other or vice versa (if the transformation is invertible). While registration using an affine transformation only compensates for global differences in pose and scaling between both images, most applications require a more flexible, non-rigid transformation to accommodate for local shape differences. While affine image registration can usually be achieved automatically and robustly in a variety of applications using maximization of mutual information [16, 17], non-rigid image registration is ill-posed due to the large number of degrees of freedom and 
ambiguity in the images, for instance in homogeneous regions. Regularization of the registration problem is therefore required to constrain the solution space to include only deformation fields that are physically acceptable and to smoothly propagate or extrapolate the registration solution from sites with salient registration features towards regions where registration clues are absent or ambiguous. One popular approach is the use of analytical basis functions to represent the deformation field, especially B-splines with local support [18]. The flexibility of the deformation and the number of degrees of freedom is controlled by the spacing between spline control points, for which a multi-resolution strategy is typically adopted. Smoothness of the deformation field is intrinsic to the parameterization at scales smaller than the control point spacing and may be imposed at larger scales by penalizing high spline curvature or excessive local volume changes. Alternatively, the deformation field can be represented as a 3D, discrete vector field that is typically regularized by adopting a physics-based elastic or viscous fluid model or a diffeomorphic deformation framework [19]. In theory, a statistical deformation model could be derived in a similar way as for landmark-based models, but this is complicated in practice by the large number of degrees of freedom in non-rigid registration, requiring a large number of examples to capture fine-scaled statistically meaningful correlations.

Image registration is frequently used in medical image analysis for inter-subject spatial normalization, for the construction of mean shape templates (atlases), for atlas-based segmentation, for quantification of local shape differences and characterization of shape variability between groups, and for spatio-temporal analysis of motion or disease evolution. Atlas-based image segmentation makes use of a prior model in the form of an atlas, typically consisting of a gray value template and associated binary or probabilistic label images, either derived from a single subject or from a mean-shape average of multiple subjects. To avoid bias in the analysis introduced by the atlas, the atlas may be stratified or specifically constructed for the specific population of subjects of interest (e.g. age, disease status). Alternatively, multiple suitable atlasses may be selected from a collection of images, each applied separately to generate a segmentation and the resulting segmentations fused, for instance using a majority voting scheme.

\subsection{Pixel classification}

Pixel classification aims at assigning an object label or its probability to each voxel in the image individually, mainly based on local intensity information alone. The classification can be supervised or unsupervised.

Model-based unsupervised classification adopts a parametric model for the expected intensities of the objects of interest, typically a Gaussian mixture model, and estimates the optimal parameters of the model and the classification simultaneously by maximizing the posterior probability of the labels given the data and the model, for instance using the Expectation-Maximization (EM) algorithm [20]. Local spatial constraints can be imposed on the classification by formulating them as a Markov 

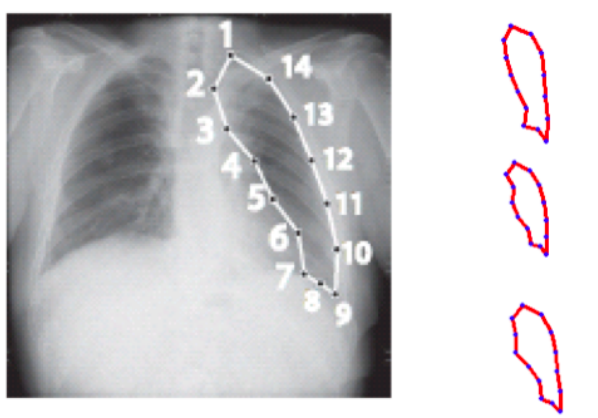

0<smiles>[Hg]</smiles><smiles>[Hg]</smiles><smiles>[CH]</smiles><smiles>[Hg]</smiles><smiles>C1=CC=C1</smiles><smiles>[CH]</smiles>
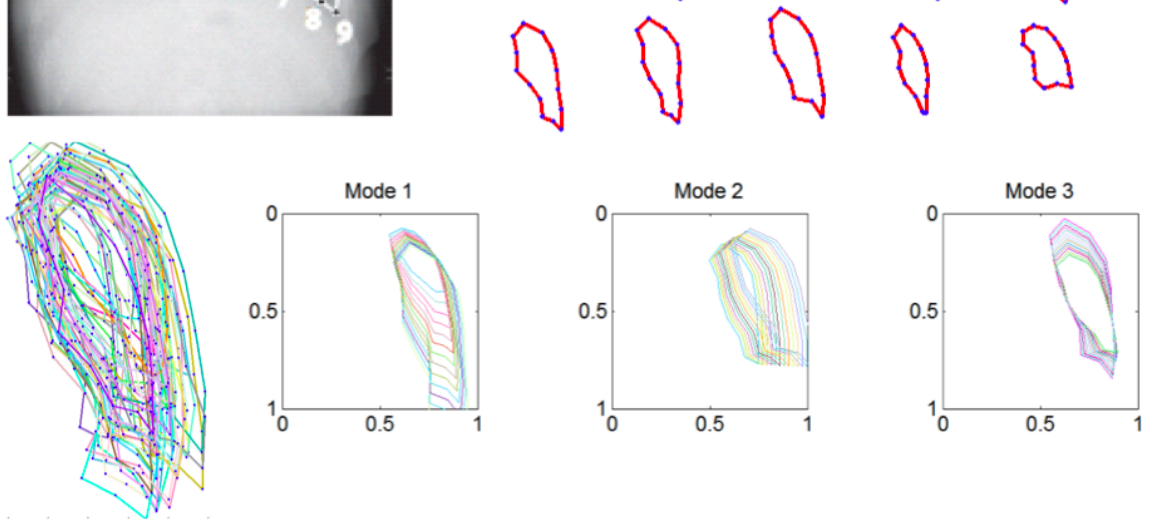

Fig. 1 Flexible shape fitting: example of a statistical shape model of the left lung in a 2D thorax radiography. The model is constructed from a set of example shapes that were manually delineated in images of different subjects. All shapes are represented by the same number of corresponding landmarks. The shapes are first aligned to eliminate irrelevant differences in pose between different subjects, after which principal component analysis is applied on the landmark coordinates to yield the mean shape and the main modes of variation around the mean. This shape model can be used for automated segmentation of the lung in new images by augmenting it with a photometric model of the local intensity patterns around each landmark.

random field. In addition, more global a priori spatial information about the objects of interest can be included as prior probability maps, typically derived from an atlas that is first registered to the images and that also serve to initiate the EM algorithm, such that the procedure becomes completely automated. Atlas-based classification and classification-based atlas registration can be unified in a single algorithm, such that both are iteratively refined. By extending this approach to the simultaneous segmentation, atlas construction and clustering of a population of multiple, possibly heterogeneous images, automated data-driven detection of morphological differences between subpopulations becomes feasible using this framework.

Intensity-based classification of pixels can be extended to more general featurebased classification of individual pixels (e.g. for segmentation) or entire images (e.g. for disease staging) whereby a vector of image-derived features, possibly augmented with non-imaging features (e.g. patient age, gender, clinical findings, genetic information ...), is computed for each pixel or per image. As the dimensionality of the feature vector increases and the features themselves are more diverse, the adoption of an underlying model for the feature distribution (e.g. multi-variate Gaussian) is 
less justified. Hence, instead of assuming a specific feature model, supervised classification methods estimate decision boundaries between different object classes in the high-dimensional feature space based on a training set of positive and negative samples of each class. Different generic classifiers can be used for this purpose, such as support vector machines, random forests or neural networks. A drawback is that suitable features need to have been defined in advance, such that the performance of these methods is impacted by feature selection and by the need for dimensionality reduction of the feature vectors to avoid overfitting in case of limited training data.

Deep learning deals with the issue of optimal feature selection by using a neural network with multiple hidden layers to learn the optimal features simultaneously while training the classifier such that overall classification performance is optimized [21]. Due to the very large number of parameters in such networks, regularization is required to avoid overfitting and to ensure generalization to unseen data. Deep neural networks, and especially convolutional neural networks, are an active topic of research in medical image analysis (see Figure 2 for an example), as these approaches currently achieve the best performance for object detection and segmentation in many applications [22].

\section{Fundamental issues}

When selecting or designing an appropriate computational strategy for model-based image analysis in a particular application, some fundamental choices or issues related to the model representation and fitting have to be considered.

\subsection{Explicit versus implicit representation of geometry}

Object shape can be described using points, contours or meshes with explicitly specified coordinates, or as a picture with implicit geometry implied by the image grid. Flexible model-to-image fitting using an explicit geometric model representation is typically driven by local photometric features along or inside the object boundary, while fitting an implicit iconic model (e.g. an atlas) involves deformable image registration. While statistical models could be built for each representation independently, unified hybrid explicit/implicit models have also been conceived in which variability in object boundary shape and iconic deformations are considered jointly, for instance by combining explicit landmark based and implicit picture based shape models in a single strategy for model-based segmentation and registration. The implicit model provides dense correspondences between a pictorial shape template and the image to be segmented by non-rigid registration constrained by statistical deformation modes. The explicit model provides point correspondences at landmark points (defined in the space of the implicit model) by flexible shape fitting constrained by a statistical model of shape variability (e.g. a PDM). While the explicit 

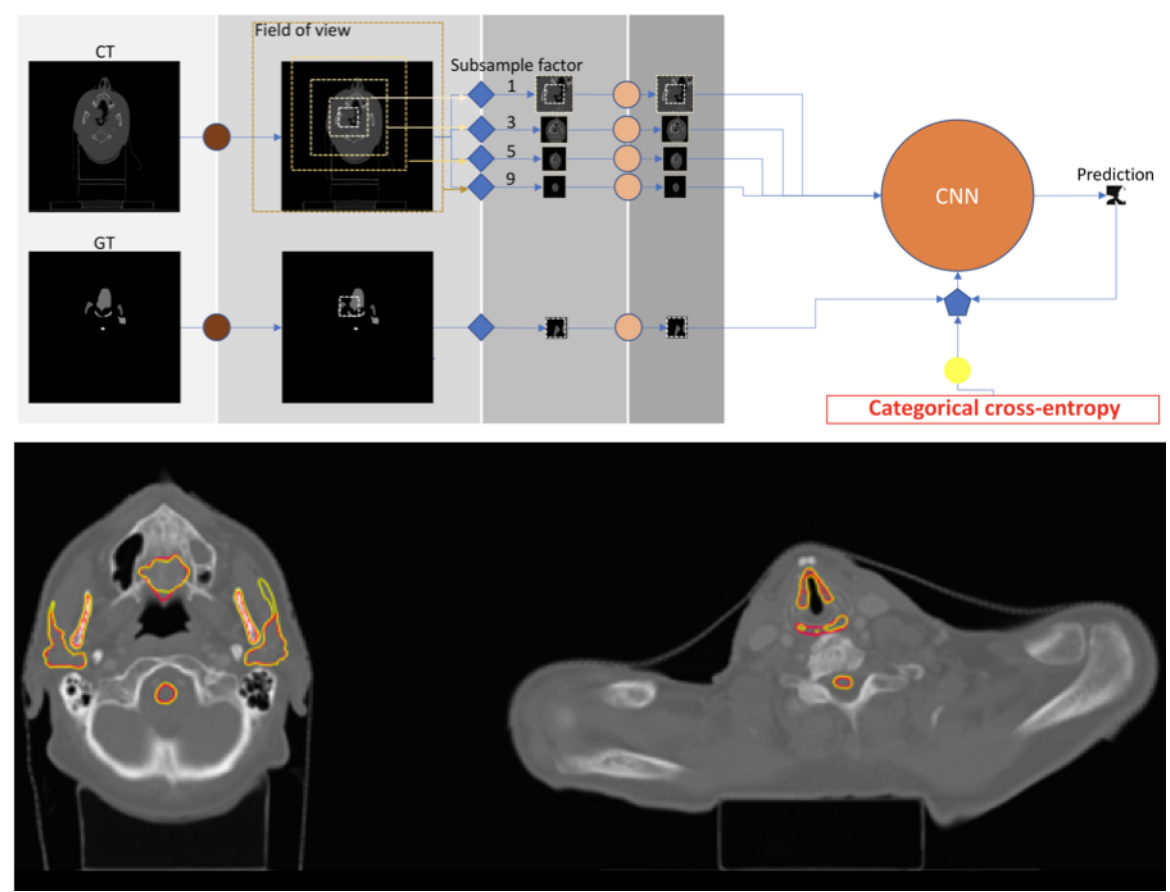

Fig. 2 Pixel classification: example of a CNN trained for organ at risk delineation in a head \& neck CT scan used for treatment planning in radiotherapy. The neural network predicts the object labels in a small patch around each voxel based on local intensity information at different scales and is trained by comparing its prediction (yellow) to the manual ground truth (red) using cross-entropy as loss function. The classifier generates delineations of multiple organs simultaneously: parotid glands, oral cavity, mandible, brainstem (left), glottic larynx, esophagus, spinal cord (right).

model is driven by intensity information at the object boundary specifically, the implicit model would bring the overall intensity appearance of the object into account. A matching strategy incorporating both models is thus expected to be more robust than each of the models separately. Moreover, by imposing that both models must be consistent at the boundary points, matching information can be propagated from one model onto the other and vice versa. Also, training of the landmark based and picture based shape models can best be performed simultaneously to exploit likely correlations between both.

\subsection{Global versus local representations of appearance}

A global model representation is based on parameters that each affect the overall (photometric and/or geometric) appearance of the object. This is advantageous for imposing global shape constraints and for propagating evidence about a suitable 
model fit from image regions with salient clues towards regions were such clues are absent. Local representations on the other hand have parameters that each describe the object appearance only locally, thus providing additional flexibility to adapt to local deviations. A unified hybrid local/global model can provide control at small scale, while at the same time imposing constraints over larger scales. Ideally, such multi-scale representation would be learned automatically from training data in order to describe the observed variability locally at the most optimal scales.

To tackle this problem, hybrid models that can represent shape variability at multiple scales are needed. For instance, in case of landmark-based shape modelling, shape constraints can be imposed between any two landmarks in a graph-like structure, both locally between adjacent landmarks as globally between more distal landmarks. The concatenation of multiple such partial statistical constraints in a single objective function may be advantageous over a single global PDM, as it requires less training samples and provides more flexibility to deal with local shape distortions. While such dependencies can be simply assumed and imposed deterministically based on application-specific heuristics, such heuristics can be avoided by learning local and global spatial correlations from the data itself. During model fitting, such dependencies need not be treated equivalently, but could be weighted to put more emphasis on shape constraints between the most correlated landmark points.

When assessing groupwise morphological variation between populations of subjects based on dense spatial correspondences established by non-rigid registration between all images, regional shape-related imaging biomarkers can be discovered based on features of interest that are derived from the deformation fields (e.g. the local Jacobian determinant of the deformation, possibly at multiple scales) obtained by a previously applied deterministic, groupwise image registration process. However, these deformation fields are likely biased by the implicit assumptions (e.g. degrees of freedom, similarity measure, regularization) made during this pre-processing stage. Such bias could be avoided by establishing dense spatial correspondences directly from the image data without having to adopt a specific deformation model.

\subsection{Deterministic versus statistical models}

Shape models in medical image analysis are often deterministic, i.e. mainly heuristic, for instance an object represented as a 2-D flexible curve with intrinsic smoothness properties, a 3-D elastic mesh, or a deformable template with physically-based deformation properties. The objective function used for fitting the model to an image is usually formulated as an energy function or similarity measure, which typically consists of a weighted combination of an external energy term, assessing the agreement of the fitted model instance with relevant photometric features derived from the image data, and one or more internal energy terms or penalty functions, imposing geometric constraints on the model. Such deterministic models are often too generic in nature and their proper behavior overly depends on the tuning of ad hoc parameters and on suitable initialization. The behavior of the model can 
be made more robust by constraining its flexibility by incorporating applicationspecific knowledge about the expected variability in image appearance of the object of interest as deduced from a representative ensemble of exemplars, i.e. a statistical appearance model. However, the initial training of such models typically relies on a deterministic approach for extracting suitable photometric features and for establishing spatial correspondences between different exemplars in the training set. The bias introduced in the model by these implicit assumptions adopted during model construction may not be ignored in case the available training data is limited. A unified hybrid deterministic/statistical modeling and model fitting approach would be able to initiate itself from small data ensembles based on generic ad-hoc assumptions that could be gradually re-assessed as the model is built up and traded for more specific knowledge about probable variability in the data as more exemplars become available.

\subsection{Data congruency versus model fidelity}

Objective functions to be optimized during model fitting typically show a trade-off between assuring fidelity of the model instance to the expected object properties versus maximizing the goodness-of-fit of the model instance to the data. Typically, with deterministic modeling approaches, this trade-off becomes apparent in the form of weight parameters that have to be set heuristically to balance the influence of different terms in the objective function in order to obtain suitable behavior of the model in a specific application. Such heuristics would not be needed if the photometric and geometric variability of the object and their interdependence would be fully modeled statistically based on joint probability models derived from actual data, instead of relying on simplifying deterministic assumptions. Model fitting is then formulated as maximizing the posterior probability of the model parameters given the observed data and their a-priori distribution. This model fitting should be robust to deviations from the expected photometric and geometric variability, due to imaging artifacts and/or pathological conditions. In practice, however, the construction of suitable priors is complicated by the limited amount of training data.

\section{Conclusion}

The evolution towards a more personalized medicine requires the analysis of a large amount of imaging (and non-imaging) data. Robust automated methods are essential for a more efficient and more accurate analysis of multi-modality images in clinical practice in the context of early diagnosis, optimal treatment planning and treatment follow-up. Medical image computing benefits from advances in machine learning to develop data-driven model-based image analysis strategies that are less biased by heuristic assumptions about the appearance of the objects in the images. Supervised 
learning using deep convolutional neural networks appears promising for various applications in medical image analysis, although the large number of parameters in these networks and the limited amount of training data available in most applications pose specific challenges.

\section{References}

[1] P. Suetens. Fundamentals of Medical Imaging, 3rd edition. Cambridge, UK: Cambridge University Press, 2017.

[2] I.N. Bankman. Handbook of Medical Imaging: Processing and Analysis. Academic Press, 2000.

[3] J.V. Hajnal, D.L.G. Hill, D.J. Hawkes. Medical Image Registration. Boca Raton, FL: CRC Press, 2001.

[4] P. Suetens, P. Fua, and A.J. Hanson. Computational strategies for object recognition. ACM Computing Surveys, 24(1):5-61, 1992.

[5] S.J.D. Prince. Computer Vision: Models, Learning, and Inference. Cambridge, UK: Cambridge University Press, 2012.

[6] H. Greenspan, B. van Ginneken, and R.M. Summers. Deep learning in medical imaging: Overview and future promise of an exciting new technique. IEEE Transactions on Medical Imaging, 35(5):1153-1159, 2016.

[7] F. Zhao and Xianghua Xie. Energy Minimization in Medical Image Analysis: Methodologies \& Applications, International Journal for Numerical Methods in Biomedical Engineering, 1-63, 2015.

[8] M. Kass, A. Witkin and D. Terzopoulos. Snakes: Active contour models. International Journal of Computer Vision, 1(4):321-331, 1988.

[9] M. Turk and A.P. Pentland. Eigenfaces for recognition. Journal of Cognitive Neuroscience, 3(1):71-96, 1991.

[10] T.F. Cootes, C.J. Taylor, D.H. Cooper, and J. Graham. Active shape models: Their training and application. Computer Vision and Image Understanding, 61(1):38-59, 1995.

[11] T.F. Cootes, G.J. Edwards, and C.J. Taylor. Active appearance models. IEEE Transactions on Medical Imaging, 23(6):681-685, 2001.

[12] S. Osher and N. Paragios. Geometric level set methods in imaging, vision, and graphics. Springer, 2003.

[13] Y. Boykov, O. Veksler, and R. Zabih. Fast approximate energy minimization via graph cuts. IEEE Transactions on pattern analysis and machine intelligence, 23(11), 1222-1239, 2001.

[14] T. Heimann and H.-P. Meinzer. Statistical shape models for 3D medical image segmentation: A review. Medical Image Analysis, 13:543-563, 2009.

[15] R. Davies, C. Twining, and C. Taylor. Statistical Models of Shape: Optimisation and Evaluation. Springer, 2008. 
[16] F. Maes, A. Collignon, D. Vandermeulen, G. Marchal, and P. Suetens, Multimodality image registration by maximization of mutual information, IEEE Trans Medical Imaging, vol. 16, pp. 187-198, 1997.

[17] J.P.W. Pluim, J.B.A. Maintz, and M.A. Viergever, Mutual-information-based registration of medical images: A survey, IEEE Transactions on Medical Imaging, 22(8): 986-1004, 2003.

[18] D. Rueckert, L.I. Sonoda, C. Hayes, D.L. Hill, M.O. Leach, and D.J. Hawkes. Nonrigid registration using free-form deformations: application to breast MR images. IEEE Transactions on Medical Imaging, 18(8), 712-721, 1996.

[19] J. Ashburner. A fast diffeomorphic image registration algorithm. Neuroimage, 38(1), 95-113, 2007.

[20] K. Van Leemput, F. Maes, D. Vandermeulen, and P. Suetens. Automated model-based tissue classiffication of MR images of the brain. IEEE Transactions on Medical Imaging, 18(10):897-908, 1999.

[21] Y. LeCun, Y. Bengio, and G.E. Hinton. Deep Learning. Nature, 521:436-444, 2015.

[22] G. Litjens, T. Kooi, B.E. Bejnordi, A.A.A. Setio, F. Ciompi, M. Ghafoorian, J.A.W.M. van der Laak, B. van Ginneken, and C.I. Snchez. A survey on deep learning in medical image analysis. Medical Image Analysis, 42:60-88, 2017. 